\title{
Reconfiguration Graph for Vertex Colourings of Weakly Chordal Graphs
}

\author{
Carl Feghali ${ }^{1 *} \quad$ Jiř́ Fiala ${ }^{2 \dagger}$ \\ ${ }^{1}$ Institutt for informatikk, \\ Universitetet i Bergen, Norway \\ 2 Department of Applied Mathematics, \\ Charles University, Prague
}

\begin{abstract}
The reconfiguration graph $R_{k}(G)$ of the $k$-colourings of a graph $G$ contains as its vertex set the $k$-colourings of $G$ and two colourings are joined by an edge if they differ in colour on just one vertex of $G$.

We show that for each $k \geq 3$ there is a $k$-colourable weakly chordal graph $G$ such that $R_{k+1}(G)$ is disconnected. We also introduce a subclass of $k$-colourable weakly chordal graphs which we call $k$-colourable compact graphs and show that for each $k$-colourable compact graph $G$ on $n$ vertices, $R_{k+1}(G)$ has diameter $O\left(n^{2}\right)$. We show that this class contains all $k$-colourable co-chordal graphs and when $k=3$ all 3 -colourable $\left(P_{5}, \overline{P_{5}}, C_{5}\right)$ free graphs. We also mention some open problems.
\end{abstract}

\section{Introduction}

Let $G$ be a graph, and let $k$ be a non-negative integer. A $k$-colouring of $G$ is a function $f: V(G) \rightarrow\{1, \ldots, k\}$ such that $f(u) \neq f(v)$ whenever $(u, v) \in E(G)$. The reconfiguration graph $R_{k}(G)$ of the $k$-colourings of $G$ has as vertex set the set of all $k$-colourings of $G$ and two vertices of $R_{k}(G)$ are adjacent if they differ on the colour of exactly one vertex (the change of the colour is the so called colour switch). For a positive integer $\ell$, the $\ell$-colour diameter of a graph $G$ is the diameter of $R_{\ell}(G)$.

In the area of reconfigurations for colourings of graphs, one focus is to determine the complexity of deciding whether two given colourings of a graph can be transformed into one another by a sequence of recolourings (that is, to decide whether there is a path between these two colourings in the reconfiguration graph); see, for example, [9, 8, 6, 4,. Another focus is to determine the diameter of the reconfiguration graph in case it is connected or the diameter of its components if it is disconnected [3, 7, 2, 5, 11]. We refer the reader to [15, 13] for excellent surveys on reconfiguration problems.

In this note, we continue the latter line of study of reconfiguration problems. In Section 3. we shall show that the $(k+1)$-colour diameter of $k$-colourable weakly chordal graphs can be infinite. On the positive side, in Section 4, we shall consider two specific subclasses of $k$-colourable perfect graphs and show that their $(k+1)$-colour diameter is quadratic in the order of the graph.

\footnotetext{
*Email: carl.feghali@uib.no, supported by the Research Council of Norway via the project CLASSIS

${ }^{\dagger}$ Email: fiala@kam.mff.cuni.cz, supported by the Czech Science Foundation (GA-ČR) project 17-09142S.
} 


\section{Preliminaries}

For a graph $G=(V, E)$ and a vertex $u \in V$, let $N_{G}(u)=\{v: u v \in E\}$. A separator of a graph $G=(V, E)$ is a set $S \subset V$ such that $G-S$ has more connected components than $G$. If two vertices $u$ and $v$ that belong to the same connected component in $G$ are in two different connected components of $G-S$, then we say that $S$ separates $u$ and $v$. A chordless path $P_{n}$ of length $n-1$ is the graph with vertices $v_{1}, \ldots, v_{n}$ and edges $v_{i} v_{i+1}$ for $i=1, \ldots, n-1$. It is a cycle $C_{n}$ of length $n$ if the edge $v_{1} v_{n}$ is also present.

The complement of $G$ is denoted $\bar{G}=(V, \bar{E})$. It is the graph on the same vertex set as $G$ and there is an edge in $G$ between two vertices $u$ and $v$ if and only if there is no edge between $u$ and $v$ in $\bar{G}$. A set of vertices in a graph is anticonnected if it induces a graph whose complement is connected. A clique or a complete graph is a graph where every pair of vertices is joined by an edge. The size of a largest clique in a graph $G$ is denoted $\omega(G)$. The chromatic number $\chi(G)$ of a graph $G$ is the least integer $k$ such that $G$ is $k$-colourable.

A graph $G$ is perfect if $\omega\left(G^{\prime}\right)=\chi\left(G^{\prime}\right)$ for every (not necessarily proper) subgraph $G^{\prime}$ of $G$. A hole in a graph is a cycle of length at least 5 and an antihole is the complement of a hole. A graph is perfect if it is (odd hole, odd antihole)-free [10]. A graph is weakly chordal if it is (hole, antihole)-free. A graph is co-chordal if it is $\left(\overline{C_{4}}\right.$, anti-hole)-free. Every weakly chordal graph is perfect. Every co-chordal graph and every $\left(P_{5}, \overline{P_{5}}, C_{5}\right)$-free graph is weakly chordal.

A 2-pair of a graph $G$ is a pair $\{x, y\}$ of nonadjacent distinct vertices of $G$ such that every chordless path from $x$ to $y$ has length 2 . We often use the following well-known lemma:

Lemma 2.1 (Hayward et al. [12]). A graph $G$ is weakly chordal graph if and only if every subgraph of $G$ is either a complete graph or it contains a 2-pair.

\section{$3 \quad$ Weakly chordal graphs}

In this section, we establish the following result.

Theorem 3.1. For each $k \geq 3$ there exists a $k$-colourable weakly chordal graph $G_{k}$ such that $R_{k+1}\left(G_{k}\right)$ is disconnected.

The graph $G_{k}$ is depicted in Figure 1 .

In other words, Theorem 3.1 states that for each $k \geq 3$ the $(k+1)$-colour diameter of $k$-colourable weakly chordal graphs can be infinite. It is worth mentioning that the case $k=2$ is already known [3] as the class of 2-colourable weakly chordal graphs is precisely the class of chordal bipartite graphs. It is also worth mentioning that Bonamy, Johnson, Lignos, Patel and Paulusma [3] asked whether the $(k+1)$-colour diameter of $k$-colourable perfect graphs is connected. This was answered negatively in [1] - the counterexample consists of a complete bipartite graph minus a matching. Our Theorem 3.1 thus strengthens this counterexample.

Proof of Theorem 3.1. It suffices to construct for each $k \geq 3$ a $k$-colourable weakly chordal graph $G_{k}$ and a $(k+1)$-colouring of $G$ such that each of the $k+1$ colours appear in the closed neighbourhood of every vertex of $G_{k}$, as then no vertex of $G_{k}$ can get recoloured.

Such graph is depicted in Figure 1. It is formed from the disjoint union of four complete graphs $K_{k-1}$, one on vertices $u_{i}$ for $i \in\{1, \ldots, k-1\}$, the other three on vertices $v_{i}, w_{i}, z_{i}$, respectively, and two further vertices $x$ and $y$. These parts are joined together by additional 


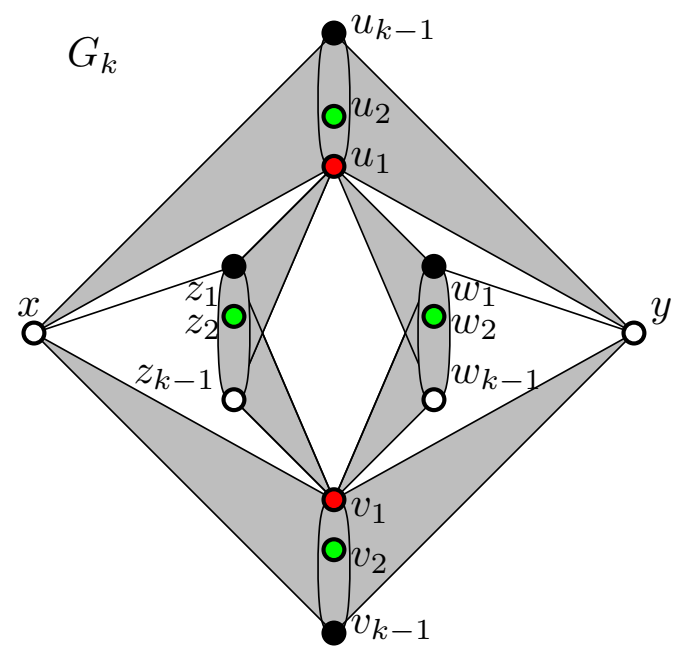

a $k$-colouring

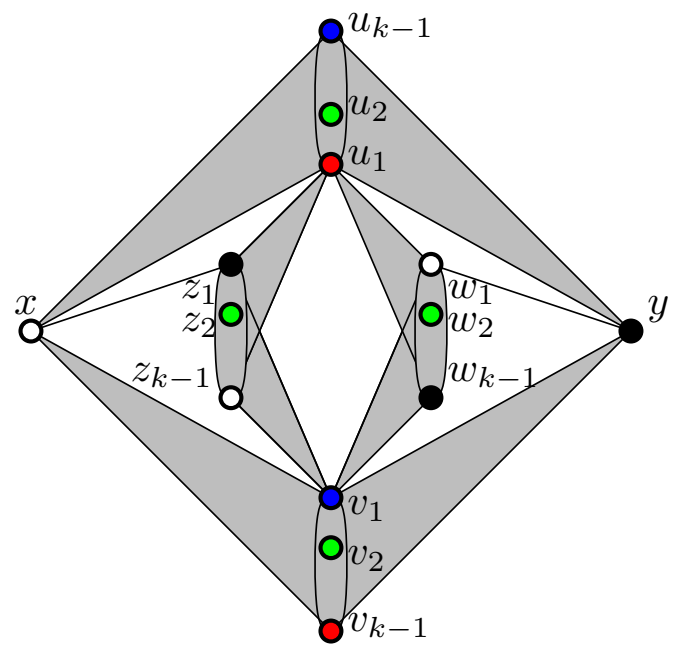

a non-switchable $(k+1)$-colouring

Figure 1: The graph $G_{k}$. Each gray area corresponds to a clique.

edges such that $x$ and $y$ are connected to each $u_{i}$ and to each $v_{i} ; u_{1}$ and $v_{1}$ are connected to each $z_{i}$ and to each $w_{i}$; and finally, $G_{k}$ contains two further edges $x z_{1}$ and $y w_{1}$.

A possible $k$-colouring of $G_{k}$ is schematically shown on the left side of Figure 1; in both pictures each 4 -tuple $u_{i}, v_{i}, w_{i}, z_{i}$ for $i \in\{2, \ldots, k-2\}$ receives a unique colour. On the other hand, in the $(k+1)$-colouring depicted on the right, every vertex of $G_{k}$ has its neighbours coloured by the $k$ remaining colours, hence no vertex can be recoloured. Hence, this colouring corresponds to an isolated vertex in the reconfiguration graph, and thus the reconfiguration graph $R_{k+1}\left(G_{k}\right)$ is disconnected.

It remains to show that $G_{k}$ is weakly chordal. Observe first that any distinct $u_{i}, u_{j}$ with $i, j \in\{2, \ldots, k-1\}$ have the same neighbourhood, hence no hole and no antihole may contain both of them. The same holds for vertices $v_{i}, w_{i}, z_{i}$, respectively. Hence without loss of generality we may assume that no vertex with index at least three participates in a hole nor in an antihole. In other words, it suffices to restrict ourselves only to the graph $G_{3}$ to show that it is weakly chordal.

By examining possible paths, one can also realise that vertices $x, y$ as well as $u_{1}, v_{1}$ form a 2-pair. Since no hole may contain a 2-pair, we may assume without loss of generality that a possible hole does not contain $y$ and $v_{1}$. The vertex $x$ separates $v_{2}$ and also the vertex $u_{1}$ separates $w_{1}, w_{2}$ from the rest in the graph $G_{3}-\left\{y, v_{1}\right\}$, hence $v_{2}, w_{1}, w_{2}$ also do not belong to a hole. We were left with five vertices $x, u_{1}, u_{2}, z_{1}, z_{2}$ which do not induce a hole, hence $G_{3}$ does not contain a hole at all.

Now assume for a contradiction that $G_{3}$ contains an antihole on at least 6 vertices. The graph $G_{3}$ contains only 6 vertices of degree at least 4 , hence the antihole contains exactly 6 vertices. The neighbourhood of $u_{2}$ induces a diamond (a $K_{4}$ minus an edge), hence it does not belong to the antihole as no diamond is an induced subgraph of $\overline{C_{6}}$. The same holds for $v_{2}, z_{2}, w_{2}$. We are left with vertices $x, y, u_{1}, v_{1}, w_{1}, z_{1}$ which do not induce an antihole in $G_{3}$, hence $G_{3}$ does not contain an antihole at all. Since $G_{3}$ is weakly chordal, we have shown that $G_{k}$ is also weakly chordal for each $k \geq 4$. 


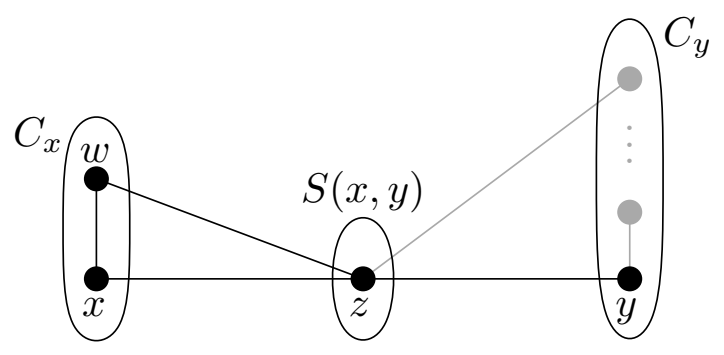

Figure 2: For the proof of Theorem 4.1. The component $C_{y}$ contains further vertices and edges outlined in gray.

\section{Quadratic diameter}

In this section, we introduce a subclass of $k$-colourable weakly chordal graphs that we call $k$-colourable compact graphs. We show in Theorem 4.1 that for each $k$-colourable compact graph $G$ on $n$ vertices the diameter of $R_{k+1}(G)$ is $O\left(n^{2}\right)$. We then show in Lemma 4.1 that $k$-colourable co-chordal graphs are $k$-colourable compact and in Lemma 4.2 that 3 -colourable $\left(P_{5}, \overline{P_{5}}, C_{5}\right)$-free graphs are 3 -colourable compact.

For a 2-pair $\{u, v\}$ of a weakly chordal graph $G$, let $S(u, v)=N_{G}(u) \cap N_{G}(v)$. Note that, by the definition of a 2-pair, $S(u, v)$ is a separator of $G$ that separates $u$ and $v$. Let $C_{v}$ denote the component of $G \backslash S(u, v)$ that contains the vertex $v$.

Definition 4.1. A weakly chordal graph $G$ is said to be compact if every subgraph $H$ of $G$ either

(i) is a complete graph, or

(ii) contains a 2-pair $\{x, y\}$ such that $N_{H}(x) \subseteq N_{H}(y)$, or

(iii) contains a 2-pair $\{x, y\}$ such that $C_{x} \cup S(x, y)$ induces a clique on at most three vertices.

Theorem 4.1. Let $k$ be a positive integer, and let $G$ be a $k$-colourable compact graph on $n$ vertices. Then $R_{k+1}(G)$ has diameter $O\left(n^{2}\right)$.

Proof. It suffices to show that we can recolour a $(k+1)$-colouring $\alpha$ of $G$ to a $(k+1)$-colouring $\beta$ by recolouring every vertex at most $2 n$ times.

We first suppose that $G$ is a complete graph. In this case, we know from [3] that we can recolour $\alpha$ to $\beta$ by recolouring every vertex at most $2 n$ times. We now consider the case when $G$ is not a complete graph but satisfies condition (ii) of compact graphs. We use induction on the number of vertices of $G$. Let $\{x, y\}$ be a 2-pair of $G$ such that $N_{G}(x) \subseteq N_{G}(y)$. From $\alpha$ and $\beta$, we can immediately recolour $x$ with, respectively, $\alpha(y)$ and $\beta(y)$. Let $G^{\prime}=G-\{x\}$, and let $\alpha_{G^{\prime}}$ and $\beta_{G^{\prime}}$ denote the restrictions of $\alpha$ and $\beta$ to $G^{\prime}$. By our induction hypothesis, we can transform $\alpha_{G^{\prime}}$ to $\beta_{G^{\prime}}$ by recolouring every vertex at most $2(n-1)$ times. We can extend this sequence of recolourings to a sequence of recolourings in $G$ by recolouring $x$ using the same recolouring as $y$. Then $x$ gets recoloured as many times as $y$ as needed.

Suppose that $G$ satisfies condition (iii) of compact graphs. We use induction on the number of vertices. If $S(x, y)$ contains exactly two vertices, then $C_{x}=\{x\}$ and hence $G$ satisfies condition (ii) of compact graphs. So we can assume that $S(x, y)$ is a single vertex $z$ 
and $C_{x}$ consists of $x$ and another vertex $w$, see Figure 2. From $\alpha$ and $\beta$, we can recolour $x$ and $w$ to another colour either immediately or by first recolouring $w$ and $x$, respectively. Let $G^{*}=G-\{x, w\}$. By our induction hypothesis, we can transform $\alpha_{G^{*}}$ to $\beta_{G^{*}}$ by recolouring every vertex at most $2(n-2)$ times. We can extend this sequence of recolourings to a sequence of recolourings in $G$ by recolouring $x$ and $w$ whenever $z$ is recoloured to their colour. At the end of the sequence we recolour $x$ and $w$ so that they agree in both colourings. As $x$ and $w$ are recoloured at most two more times as $z$, this completes the proof.

Lemma 4.1. Every $k$-colourable co-chordal graph is compact.

Proof. Let $G$ be a $k$-colourable co-chordal graph. If $G$ is a complete graph, then $G$ is compact by definition. Otherwise, since $G$ is weakly chordal, $G$ contains a 2-pair $\{x, y\}$ by Lemma 2.1 . If $x$ has a neighbour $x_{1}$ that is not a neighbour of $y$ and $y$ has a neighbour $y_{1}$ that is not a neighbour of $x$, then $x_{1}$ is not adjacent to $y_{1}$, as otherwise $S(x, y)$ does not separate $x$ and $y$. But then the edges $x x_{1}$ and $y y_{1}$ form $\overline{C_{4}}$, a contradiction. Therefore, $N_{G}(x) \subseteq N_{G}(y)$ or vice-versa and hence the graph $G$ is compact as required.

Lemma 4.2. Every 3-colourable $\left(P_{5}, \overline{P_{5}}, C_{5}\right)$-free graph is compact.

The proof of this lemma will require a little more work. First, we need some definitions and auxiliary results. When $T$ is a set of vertices of a graph $G$, a set $D \subseteq V(G) \backslash T$ is $T$-complete if each vertex of $D$ is adjacent to each vertex of $T$. Let $D(T)$ denote the set of all $T$-complete vertices.

Lemma 4.3 (Trotignon and Vušković [14]). Let $G$ be a weakly chordal graph, and let $T \subseteq$ $V(G)$ be a set of vertices such that $G[T]$ is anticonnected and $D(T)$ contains at least two non-adjacent vertices. If $T$ is inclusion-wise maximal with respect to these properties, then any chordless path of $G \backslash T$ whose ends are in $D(T)$ has all its vertices in $D(T)$.

The following corollary is implicit in [14.

Corollary 4.1. Let $G$ be a weakly chordal graph that contains a chordless path $P$ of length 2 . Then there exists an anticonnected set $T$ containing the centre of $P$, such that $D(T)$ contains a 2-pair of $G$.

In particular, the 2-pair can always be found in the neighbourhood of the centre of $P$.

Proof. We start with the centre of $P$ to build our set $T$ as in Lemma 4.3. Then $D(T)$ is not a clique as it contains both ends of $P$. Hence, by definition of weakly chordal graphs, $D(T)$ contains a 2-pair. This 2-pair is also a 2-pair of $G$ by Lemma 4.3 .

We are now ready to prove Lemma 4.2 .

Proof of Lemma 4.2. Let $G$ be a 3 -colourable $\left(P_{5}, \overline{P_{5}}, C_{5}\right)$-free graph. Suppose towards a contradiction that $G$ is not compact. In particular, $G$ is not a complete graph, as otherwise $G$ would be compact by the definition. Since $G$ is weakly chordal, it contains a 2-pair $\{x, y\}$. We choose $\{x, y\}$ such that $\left|V\left(C_{x}\right)\right|$ is minimum over all 2-pairs $\{x, y\}$ of $G$.

Denote by $G^{\prime}$ the subgraph of $G$ induced by the union of $S(x, y)$ and the vertices of $C_{x}$. The subgraph $G^{\prime}$ is not complete, as $G$ would be compact, hence $G^{\prime}$ contains a chordless path of length 2. Let us argue that $G^{\prime}$ contains a chordless path of length 2 whose centre is, in fact, a member of $C_{x}$. 

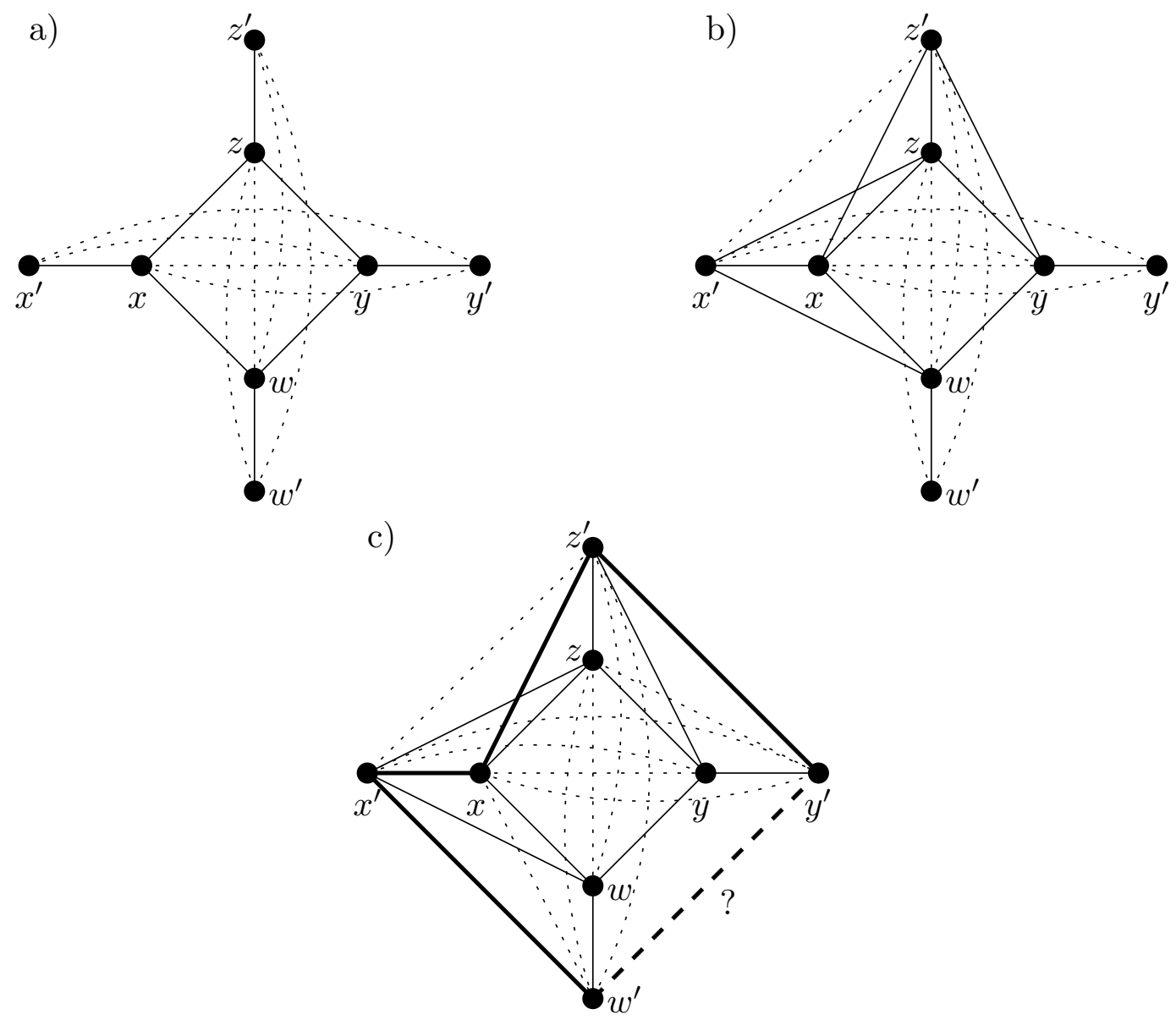

Figure 3: The case analysis for the proof of Lemma 4.2. The dotted connections indicate nonadjacent vertices.

If $C_{x}$ is not complete, then this is immediate. And if $S(x, y)$ is not complete, then it contains a pair of vertices $u$ and $v$ that are not adjacent, so we take $u, x, v$ to be our path. Hence we can assume that $C_{x}$ and $S(x, y)$ are both complete and, as $G^{\prime}$ is not complete, there must be a vertex $u$ of $C_{x}$ and a vertex $v$ of $S(x, y)$ such that $u v \notin E(G)$. Then we can take $u, x, v$ as our path and our aim is achieved.

Applying Corollary 4.1 with $P$ being a chordless path of length 2 whose centre is in $C_{x}$, we find that $G^{\prime}$ contains a 2-pair $\{z, w\}$ that is also a 2-pair of $G$.

We next want to argue that $z, w \in S(x, y)$. For a contradiction, assume without loss of generality that $z$ belongs to $C_{x}$. This implies that $S(z, w) \subseteq V\left(G^{\prime}\right)$. So there must be a component $C_{1}$ of $G \backslash S(z, w)$ such that $C_{1}$ and $S(x, y)$ do not have a vertex in common since $y$ is adjacent to every vertex of $S(x, y)$. Therefore, we find that $C_{1} \subseteq C_{x}$. If $C_{1}=C_{x}$, then $S(x, y)=S(z, w)$ and hence $z, w \in C_{1}$ which is impossible because $S(z, w)$ separates $z$ and $w$. Therefore, $\left|V\left(C_{1}\right)\right|<\left|V\left(C_{x}\right)\right|$ holds, which contradicts our choice of $C_{x}$.

Hence we have concluded that $z, w \in S(x, y)$. Now the vertices $x, z, y, w$ form a cycle such that $\{x, y\}$ and $\{z, w\}$ are 2-pairs. Since $G$ is not compact, $N_{G}(x) \nsubseteq N_{G}(y)$, hence there exists a vertex $x^{\prime}$ that is adjacent to $x$ but not to $y$. Analogously, there are vertices $y^{\prime}, z^{\prime}, w^{\prime}$ 


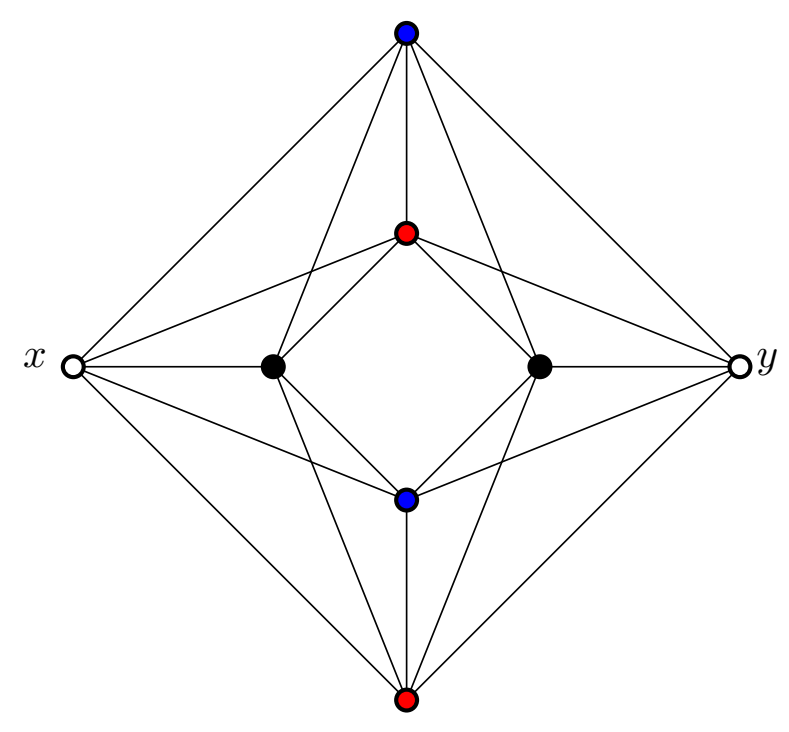

Figure 4: $\mathrm{A}\left(P_{5}, \overline{P_{5}}, C_{5}\right)$-free 4 -colourable graph $H$ that is not compact.

such that $y y^{\prime}, z z^{\prime}, w w^{\prime} \in E$, but $x y^{\prime}, w z^{\prime}, z w^{\prime} \notin E$. If $x^{\prime}=z^{\prime}$, then $x^{\prime}$ must be adjacent to $y$ or $w$ else $x^{\prime}, x, z, y, w$ form $\overline{P_{5}}$. But $x^{\prime}$ is not adjacent to $y$ and $z^{\prime}$ not adjacent to $w$, thus $x^{\prime} \neq z^{\prime}$. Similarly, $y^{\prime} \neq w^{\prime}$ and hence $x^{\prime}, y^{\prime}, w^{\prime}, z^{\prime}$ are distinct. Moreover, since $S(x, y)$ is a separator, we get $x^{\prime} y^{\prime} \notin E$ and analogously $w^{\prime} z^{\prime} \notin E$, see Figure 3 a).

If both $z^{\prime}$ and $w^{\prime}$ are not adjacent to $x$, then $z^{\prime}, z, x, w, w^{\prime}$ form $P_{5}$. So we can assume without loss of generality that $z^{\prime}$ is adjacent to $x$. If $z^{\prime}$ is not adjacent to $y$, then $z^{\prime}, z, x, w, y$ would form $\overline{P_{5}}$ as $w$ is not adjacent to $z^{\prime}$. Thus $z^{\prime}$ is adjacent to both $x$ and $y$.

Similarly, to avoid $P_{5}$ on vertices $x^{\prime}, x, z, y, y^{\prime}$ either $x^{\prime}$ or $y^{\prime}$ must be adjacent to $z$ and hence also to $w$, so we assume without loss of generality that $x^{\prime}$ is adjacent to $z$ and $w$. If $x^{\prime}$ is adjacent to $z^{\prime}$, then $x, z, z^{\prime}, x^{\prime}$ form $K_{4}$, a contradiction with the assumption the $G$ is 3-colourable, see Figure 3 b).

To avoid a $P_{5}$ on the vertices $x^{\prime}, x, z^{\prime}, y, y^{\prime}$, the vertices $z^{\prime}$ and $y^{\prime}$ are forced to be adjacent. Similarly $x^{\prime} w^{\prime} \in E$ as otherwise $z^{\prime}, z, x^{\prime}, w, w^{\prime}$ induce a $P_{5}$. To avoid a $K_{4}$ on the vertices $z, z^{\prime}, y, y^{\prime}$, the vertices $z$ and $y^{\prime}$ need to be nonadjacent. Similarly $x w^{\prime} \notin E$ as otherwise $x, x^{\prime}, w, w^{\prime}$ induce a $K_{4}$.

Now the vertices $w^{\prime}, x^{\prime}, x, z^{\prime}, y^{\prime}$ induce either a $C_{5}$ or a $P_{5}$, depending whether the edge $y^{\prime} w^{\prime}$ is present or not, see Figure 3 c). In either case we arrive at a contradiction and the lemma is proved.

We are aware the concept of compact graphs does not fit tight with the class of $\left(P_{5}, \overline{P_{5}}, C_{5}\right)$ free graphs, as some of these graphs need not to be $k$-colourable compact graphs for $k \geq 4$. An example of such graph $H$ for $k=4$ is depicted in Figure. 4 .

Due to symmetries of the graph $H$ it suffices without loss of generality to consider only the 2-pair $\{x, y\}$ as other 2-pairs could be mapped onto $\{x, y\}$ by an automorphism of $H$. Observe that this 2-pair violates the conditions of the definition 4.1 for $H$ to be 4-colourable compact.

Any choice of five vertices from $H$ would contain two vertices joined by a horizontal or a vertical edge, and such edge cannot be extended to an induced $P_{3}$, hence $H$ is also $P_{5}$-free. 
Also, such choice of five vertices would contain two opposite vertices either of the inner $C_{4}$ or from the outer one, like the vertices $x$ and $y$. As such two vertices form an 2-pair, $H$ contains no $C_{5}$. Finally, $H$ has only two induced $C_{4}$ and neither could be completed by any fifth vertex to a $\overline{P_{5}}$.

\section{Concluding remarks}

We end this note with two open problems.

Problem 1. For which integer $\ell>k+1$ is the $\ell$-colour diameter of $k$-colourable weakly chordal graphs connected?

Problem 2. Is it true that the $(k+1)$-colour diameter of $k$-colourable $\left(P_{5}, \overline{P_{5}}, C_{5}\right)$-free graphs is quadratic for each $k \geq 4$ ?

\section{References}

[1] M. Bonamy and N. Bousquet. Recoloring graphs via tree decompositions. arXiv, 1403.6386, 2014.

[2] M. Bonamy and N. Bousquet. Recoloring graphs via tree decompositions. European Journal of Combinatorics, 69:200-213, 2018.

[3] M. Bonamy, M. Johnson, I. M. Lignos, V. Patel, and D. Paulusma. Reconfiguration graphs for vertex colourings of chordal and chordal bipartite graphs. Journal of Combinatorial Optimization, 27:132-143, 2014.

[4] P. Bonsma and L. Cereceda. Finding paths between graph colourings: PSPACEcompleteness and superpolynomial distances. Theoretical Computer Science, 410(50):5215-5226, 2009.

[5] N. Bousquet and G. Perarnau. Fast recoloring of sparse graphs. European Journal of Combinatorics, 52:1-11, 2016.

[6] R. C. Brewster, S. McGuinness, B. Moore, and J. A. Noel. A dichotomy theorem for circular colouring reconfiguration. Theoretical Computer Science, 639:1-13, 2016.

[7] L. Cereceda, J. van den Heuvel, and M. Johnson. Connectedness of the graph of vertexcolourings. Discrete Mathematics, 308:913-919, 2008.

[8] L. Cereceda, J. van den Heuvel, and M. Johnson. Mixing 3-colourings in bipartite graphs. European Journal of Combinatorics, 30(7):1593-1606, 2009.

[9] L. Cereceda, J. van den Heuvel, and M. Johnson. Finding paths between 3-colourings. Journal of Graph Theory, 67(1):69-82, 2011.

[10] M. Chudnovsky, N. Robertson, P. Seymour, and R. Thomas. The strong perfect graph theorem. Annals of mathematics, pages 51-229, 2006.

[11] C. Feghali, M. Johnson, and D. Paulusma. A reconfigurations analogue of Brooks' theorem and its consequences. Journal of Graph Theory, 83(4):340-358, 2016. 
[12] R. Hayward, C. Hoàng, and F. Maffray. Optimizing weakly triangulated graphs. Graphs and Combinatorics, 5(1):339-349, 1989.

[13] N. Nishimura. Introduction to reconfiguration. Algorithms, 11(4):52, 2018.

[14] N. Trotignon and K. Vušković. On Roussel-Rubio-type lemmas and their consequences. Discrete Mathematics, 311(8-9):684-687, 2011.

[15] J. van den Heuvel. The complexity of change. Surveys in Combinatorics 2013, edited by S. R. Blackburn, S. Gerke, and M. Wildon, London Mathematical Society Lecture Notes Series, 409, 2013. 